\title{
Electron Transport in a Double Quantum Dot Governed by a Nuclear Magnetic Field
}

\author{
Oleg N. Jouravlev* and Yuli V. Nazarov \\ Kavli Institute of NanoScience, Delft University of Technology, Lorentzweg 1, 2628 CJ Delft, The Netherlands
}

(Received 28 July 2005; published 5 May 2006)

\begin{abstract}
We investigate theoretically electron transfer in a double dot in a situation where spin blockade is lifted by nuclear magnetic field: this has been recently achieved in experiment [F. Koppens et al., Science 309, 1346 (2005)]. We show that for a given realization of nuclear magnetic field spin blockade can be restored by tuning external magnetic field; this may be useful for quantum manipulation of the device.
\end{abstract}

PACS numbers: 85.35.Be, 71.70.Jp, 73.23.-b

Much modern research is devoted to practical realization of quantum manipulation and computation (QMC). Although QMC can be performed with convenient magnetic resonance techniques [1], this necessarily involves macroscopically many identical spins. The challenge is to do QMC with individual spin states, e.g., those of localized electrons. Remarkable experimental progress has been recently achieved in preparation, manipulation, and measurement of individual spin quantum states in quantum dots. An important issue to resolve in this case is the spin measurement: to do this, one has to convert spin into charge and/or electric current $[2,3]$. Such conversion has been realized in a single quantum dot [4,5]. Other experiments were focused on the transport through two coupled quantum dots [6,7]. Although such double dot is a more complicated system with many additional processes influencing spin and charge transfer, the advantage is an immediate access to spin-charge conversion. In a double dot, two electron spins can be entangled in the course of quantum manipulation forming either symmetric spin singlet or antisymmetric triplet states. This strongly affects electron transport giving rise to spin blockade of electron tunneling [8]. The quantum dots are commonly fabricated in GaAsbased semiconductor heterostructures. The specifics of GaAs is a strong hyperfine interaction between electron and nuclear spins [9]. Therefore, the spin of an electron localized in a quantum dot can be strongly affected by the effective spin magnetic field $\boldsymbol{B}^{N}$ arising from random configuration of many nuclear spins situated in the dot. This field helps the transitions between the components of spin doublet [10] as well as between singlet and triplet states [11]. It has been experimentally proven that the nuclear field not only lifts spin blockade in a double dot but gives rise to time-dependent spin-driven oscillations of the current [12]. Similar effects have been also observed in quantum Hall constriction [13]. The origin of the oscillations is the modulation of the current by nuclear field and feedback of electron spin on nuclear spins that results in their nutations [14]. Since the nuclear field is random and hardly controllable, its influence on the electron spin significantly complicates QMC. This has motivated intensive research aimed to measure and to predict the effect of the nuclei on spins in quantum dots $[6,7,15]$.
The study presented here has been stimulated by very recent experiment in this direction [15]. The advantage achieved in this experiment is the better control of electron levels in the dots and, most importantly, the possibility to control and tune the tunnel coupling between dots in a wide range. Most interesting results were obtained near the boundary of Coulomb diamond where the states that differ by number of electrons in the dot are aligned in energy. The authors of [15] were able to demonstrate the order-of-value change of the current by anomalously small external magnetic field $\boldsymbol{B} \simeq 5 \mathrm{mT}$ that matches the nuclear field. By tuning the tunnel coupling, they observe this effect in several different regimes.

We provide an adequate theoretical framework for this experimental situation. We derive and solve density matrix equation valid in the regimes of interest. We concentrate on the fact that nuclear field randomly changes at time scale bigger than that of electron dynamics but smaller than the measurement time and therefore provides "frozen disorder" for electron spins. We achieve agreement with experiment in rather fine detail. A very important result of our analysis is that for any given configuration of nuclear magnetic field there is always a value of external magnetic field at which there is no current-stopping point. This encourages us to speculate that the fast current measurement in this setup can be used to measure and, via external feedback, partly compensate the nuclear magnetic field. The setup would be stabilized in the stopping point where spin blockade is restored and QMC is possibly enabled.

The charge configuration of the double dot is given by the number of electrons in the left and right dot $\left(N_{L}, N_{R}\right)$. The gate and bias voltages are tuned in the experiment to provide the following transport cycle: $(1,1) \rightarrow$ $(0,2) \rightarrow^{\Gamma_{R}}(0,1) \rightarrow^{\Gamma_{L}}(1,1)$. Two last processes are incoherent tunnel transitions with electron transfer from the left and to the right lead, the tunneling rates being $\Gamma_{L}$ and $\Gamma_{R}$, respectively. The first transition may be coherent and is due to tunnel coupling $t$ between electron states in different dots. If spin is taken into account, there are four possible quantum states in $(1,1)$ configuration: a singlet $S(1,1)$ and there components of the triplet $T_{i}(1,1)$. As to $(0,2)$ configuration, only a singlet state $S_{g}(0,2)$ participates in the transport: the triplet states of $(0,2)$ are much higher in 
energy owing to strong exchange interaction between two electrons in the same dot. The essence of spin blockade is the spin selection rule for $(1,1) \rightarrow(0,2)$. Provided spin is conserved, there is no matrix element connecting any triplet state $T(1,1)$ and $S_{g}(0,2)$. Therefore, the transition does not take place, the system gets stuck in one of the triplet states and the current is blocked.

The part of the Hamiltonian for $(1,1)$ and $(0,2)$ configurations that conserves spin is presented in the triplet-singlet states basis $\left(T_{i}, S\right.$ and $\left.S_{g}\right)$ as

$$
\begin{aligned}
\hat{H}_{0}= & E\left(|S\rangle\left\langle S\left|+\sum_{i}\right| T_{\mathrm{i}}\right\rangle\left\langle T_{\mathrm{i}}\right|\right)+(E+\Delta)\left|S_{g}\right\rangle\left\langle S_{g}\right| \\
& +t\left(|S\rangle\left\langle S_{g}|+| S_{g}\right\rangle\langle S|\right) .
\end{aligned}
$$

Here $\Delta$ is the detunning of $(1,1)$ and $(0,2)$ states linear in the gate and bias voltages. The experiments were concentrated at the edge of the Coulomb blockade diamond where $|\Delta| \ll e V, E_{C}$. The tunnel coupling between the dots mixes two singlets at $|\Delta| \simeq t$ but does not alter triplet states (Fig. 1).

The leakage current in spin blockade regime can only arise from the spin-dependent interactions that mix singlet and triplet states. Theoretically, such interactions can be caused by many mechanisms [16]. Experimentally, the most relevant one appears to be hyperfine interaction with nuclear spins. Since there are many nuclear spins interacting with an electron state in each dot, their net effect can be presented in terms of classical variables: effective fields $\boldsymbol{B}_{L, R}^{N}$ (we measure fields in energy units). In the absence of net nuclear polarization, these fields are random depending on a concrete configuration of nuclear spins

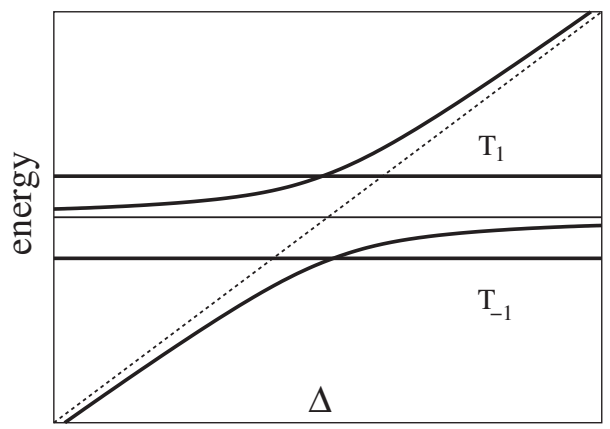

FIG. 1. Energies of the states with two electrons. The tunnel coupling between the dots mixes singlet states $S, S_{g}$ and does not influence triplet states (split by magnetic field).

[10]. Owing to central limit theorem, the distribution of both fields is Gaussian with $\left\langle\left\langle\left(\boldsymbol{B}_{L, R}^{N}\right)^{2}\right\rangle\right\rangle \equiv B_{N}^{2}=E_{n}^{2} / N_{\text {eff }}$, $E_{n} \approx 0.135 \mathrm{meV}$ for GaAs being the energy splitting induced by fully polarized nuclei, $N_{\text {eff }}$ being the effective number of nuclei in the dot, $N_{\text {eff }} \simeq 10^{5-6}$ for typical dots. It is important for our approach that nuclear fields change at time scale of nuclear spin relaxation $(\simeq 1 \mathrm{~s})$, that is much bigger than any time scale associated with electron transport. This is why they can be regarded as stationary random fields. The electron spins inside the dots feel therefore effective stationary fields described by

$$
\hat{H}_{\text {spin }}=\boldsymbol{B}_{L}^{N} \cdot \boldsymbol{S}_{L}+\boldsymbol{B}_{R}^{N} \cdot \boldsymbol{S}_{R}+B\left(S_{L}^{z}+S_{R}^{z}\right),
$$

$S_{L, R}$ being the operators of the electron spin in each dot and the external magnetic field is $\| z$. We rewrite this in tripletsinglet representation as

$$
\hat{H}_{\text {spin }}=B_{s}^{z} \sum_{i} s_{z}^{i}\left|T_{\mathrm{i}}\right\rangle\left\langle T_{\mathrm{i}}\left|+B_{a}^{z}\right| S\right\rangle\left\langle T_{0}\right|+\sum_{ \pm}\left(\frac{B_{s}^{x} \pm i B_{s}^{y}}{\sqrt{2}}\left|T_{0}\right\rangle\left\langle T_{ \pm 1}\left|+\frac{\mp B_{a}^{x}-i B_{a}^{y}}{\sqrt{2}}\right| S\right\rangle\left\langle T_{ \pm 1}\right|+\text { H.c. }\right) \text {, }
$$

where $\quad \boldsymbol{B}_{a}=\left(\boldsymbol{B}_{L}^{N}-\boldsymbol{B}_{R}^{N}\right) / 2, \quad \boldsymbol{B}_{s}=\left(\boldsymbol{B}_{L}^{N}+\boldsymbol{B}_{R}^{N}\right) / 2+B z$, and $s_{z}^{i}=-1,0,1$ is the projection of the spin of $\left|T_{i}\right\rangle$ state on $z$ axis. We see that the sum of effective fields mixes and splits triplet components only. The difference of the fields mixes the spin singlet $S(1,1)$ and triplet $T(1,1)$ states, this being the source of leakage current.

The energy levels of the resulting Hamiltonian $\hat{H}_{s t}=$ $\hat{H}_{0}+\hat{H}_{\text {spin }}$ are determined now not only by the tunneling $t$ and misalignment of the levels $\Delta$ but also by the fields, the corresponding energy scales can be comparable. The mixing of the singlet and triplet in the eigenstates of the Hamiltonian can be significant as well. Already from analysis of this simple Hamiltonian we can conclude that the current is absent if either $\boldsymbol{B}_{s} \| \boldsymbol{B}_{a}$ or $\boldsymbol{B}_{s} \perp \boldsymbol{B}_{a}$, since here $\boldsymbol{B}_{s}$ consists of the external and sum of nuclear magnetic fields. To see this explicitly from (3), let us choose $z$ axis in the direction of $\boldsymbol{B}_{s}$. If $\boldsymbol{B}_{s} \| \boldsymbol{B}_{a}$, two triplet states $\left|T_{ \pm 1}\right\rangle$ are not mixed with the singlet. If $\boldsymbol{B}_{s} \perp \boldsymbol{B}_{a}$, it is one state $\left|T_{0}\right\rangle$ that is not mixed. In both cases the system sticks in one of the nonmixed triplet states resulting in no current.
Importantly, the stopping point $\boldsymbol{B}_{s} \perp \boldsymbol{B}_{a}$ can be achieved at any configuration of nuclear fields by adjusting the external field $B$.

To evaluate the current in general situation, we proceed with formulation of a suitable density matrix approach first elaborated for double quantum dot in [17]. Current for the transport cycle given is proportional to the probability to find a system in the state $S_{g}, I=e \Gamma_{\mathrm{R}} \rho_{\mathrm{S}_{g} S_{g}}$. Although the transport involves 7 states, the probabilities of $(1,0)$ doublets are readily expressed via other probabilities. So the density matrix to work with is spanned by five singlettriplet states discussed. Using the equations of motion, we derive the equations for the stationary density matrix $(d \hat{\rho} / d t=0)$. Five diagonal equations read

$$
\begin{array}{r}
\frac{1}{4} \Gamma_{R} \rho_{\mathrm{S}_{g} S_{g}}-i\left\langle T_{i}\left|\left[\hat{H}_{\mathrm{s}}, \hat{\rho}\right]\right| T_{i}\right\rangle=0, \\
\frac{1}{4} \Gamma_{R} \rho_{\mathrm{S}_{g} S_{g}}-\Gamma_{\mathrm{in}} \rho_{\mathrm{SS}}-i\left\langle S\left|\left[\hat{H}_{\mathrm{st}}, \hat{\rho}\right]\right| S\right\rangle=0, \\
-\Gamma_{R} \rho_{\mathrm{S}_{g} S_{g}}+\Gamma_{\mathrm{in}} \rho_{\mathrm{SS}}-i\left\langle S_{g}\left|\left[\hat{H}_{\mathrm{st}}, \hat{\rho}\right]\right| S_{g}\right\rangle=0,
\end{array}
$$

where, motivated by experiment, we also include inelastic 
transitions between $S$ and $S_{g}$ with the rate $\Gamma_{\text {in }}, \Gamma_{\text {in }} \neq 0$ if $\Delta<0$. The commutator terms include nondiagonal elements of density matrix, so we also need 20 nondiagonal equations,

$$
-\frac{1}{2}\left(\Gamma^{j}+\Gamma^{i}\right) \rho_{\mathrm{jk}}-i\left\langle j\left|\left[\hat{H}_{\mathrm{st}}, \hat{\rho}\right]\right| k\right\rangle=0 .
$$

Here $j, k=T_{i}, S, S_{g}$ number the five states basis, $j \neq k$. The "rates" $\Gamma_{j}$ are zero for triplet states and are $\Gamma_{R}\left(\Gamma_{\text {in }}\right)$ for $S_{g}(S)$. To close the set of equations we use the normalization condition for density matrix, $\sum \rho_{j j}+\rho_{\mathrm{S}_{g} S_{g}}(1+$ $\left.\Gamma_{R} / \Gamma_{L}\right)=1, j=T_{i}, S$.

The solution gives the current for a given realization of nuclear fields. Normally, one expects self-averaging over different realizations at time scale of a single measurement. Since nuclear relaxation times are large, this point deserves some discussion. In fact, raw data acquisition time in experiment [15] was $0.1 \mathrm{~s}$ per point, which is probably less than the relaxation time. However, the raw data are noisy (see Fig. 4) due to both instrumental noise and random changes of nuclear fields. An accurate measurement requires, say, 50 data points, this takes time much bigger than the relaxation time. This leads us to two conclusions: (i) smooth part of experimental data corresponds to the current averaged over realizations, (ii) a realistic (factor of 30) improvement of the measurement speed and accuracy will allow us to measure current for a given realization. So that, to compare our theory with experimental results, we average the current obtained from the solution of equation set (4) and (5) over Gaussian distribution of fields.

Both solving and averaging can be easily done numerically. To present the physics behind this, we give analytical results in two limiting cases. In both limiting cases, the tunneling via the left junction is much faster than through the right one, so that the results do not depend on $\Gamma_{L}$. The first, natural limit corresponds to small nuclear fields, $B_{N} \ll \max (t,|B|)$. In this case, the system is preferentially in one of the triplet states whose energies are $0, \pm B_{s}$. It is convenient in this case to choose spin quantization axis along $\boldsymbol{B}_{s}$ and work with parallel and perpendicular components of $\boldsymbol{B}_{a}, B_{a}^{\|, \perp}$ with respect to this axis. The current reads

$$
\begin{aligned}
\Gamma_{R} e / I & =\left(\frac{t^{2}}{\left(B_{a}^{\|}\right)^{2}}+\frac{F\left(B_{s}\right)}{\left(B_{a}^{\perp}\right)^{2}}\right) ; \\
F\left(B_{s}\right) & =t^{2}+B_{s}^{2}\left[2+\left(B_{s}^{2}+\Delta^{2}\right) / t^{2}\right],
\end{aligned}
$$

where the first term is due to transitions from $\left|T_{0}\right\rangle$ and the second due to transitions from $\left|T_{ \pm 1}\right\rangle$. As expected, the current stops if either $B_{a}^{\|}=0$ or $B_{a}^{\perp}=0$. The average current in this limit is

$$
I / e \Gamma_{R}=\left\{\begin{array}{ll}
B_{N}^{2} / 15 t^{2} & B \rightarrow 0 \\
B_{N}^{2} t^{2} / 3 B^{4} & |B| \rightarrow \infty
\end{array} .\right.
$$

We plot the results in this limit for average current as well as for two arbitrary realizations of the field (Fig. 2). The stopping points at $B \simeq B_{N}$ are visible for realizations, while no features in average current are seen in this range.

The alternative limit of big fields is achieved provided $|B|, B_{N} \gg \Delta_{\mathrm{ST}} \equiv \max \left(t, t^{2} /|\Delta|\right)$. In this case, the system sticks in one of the four states $(1,1)$ with energies $\pm\left(B_{L} \pm\right.$ $\left.B_{R}\right)$. The current is determined by decay from these states and reads

$$
I / e=\Gamma_{R} \frac{t^{2}\left(\boldsymbol{n}_{L} \times \boldsymbol{n}_{R}\right)^{2}}{8\left(\Delta^{2}+B_{s}^{2}\right)},
$$

where $\boldsymbol{n}_{L, R}$ are unit vectors in the direction of $\boldsymbol{B}_{L, R}$. At $B=0 I / e \simeq \Gamma_{R} t^{2} / B_{N}^{2}$ and it drops significantly at $|B| \simeq$ $\max \left(B_{N},|\Delta|\right)$.

Equation (8) seemingly contradicts our general statement giving nonzero current at $\boldsymbol{B}_{s} \perp \boldsymbol{B}_{a}$. A fine point is that Eq. (8) is not valid in close vicinity stopping point where two of the four states are degenerate. One has to take into account that this degeneracy is lifted by coupling to $S_{g}$. As a result, the current develops a narrow Lorenzianshaped dip in the vicinity of the stopping point,

$I / I_{0}=\frac{\left(\delta B / B_{w}\right)^{2}}{1+\left(\delta B / B_{w}\right)^{2}} ; \quad \delta B=B-B^{(0)}, \quad B_{w} \simeq \frac{t^{2}}{\Delta}$.

Since the dip is narrow (see Fig. 3), it is washed away upon averaging.

The average current

$$
I / e \Gamma_{R}= \begin{cases}t^{2} B_{N}^{2} / 6 B^{2}\left(\Delta^{2}+B^{2}\right) & |\Delta|,|B| \gg B_{N} \\ t^{2} / 12 \Delta^{2} & |\Delta| \gg B_{N} \gg|B| . \\ \text { const } \times t^{2} / B_{N}^{2} & |\Delta|,|B| \ll B_{N}\end{cases}
$$

We have disregarded $\Gamma_{\text {in }}$ in the above estimations assuming it is smaller than the rates estimated. We encounter a similar situation under opposite conditions. We assume now that the four states are predominantly emptied by inelastic tunneling, $\Gamma_{\text {in }} \gg \Gamma_{R}(t / \Delta)^{2}$, while their splitting is determined by magnetic field, $B_{N} \gg \Delta_{\mathrm{ST}}$. It is experimentally confirmed that this always takes place at sufficiently big negative $\Delta$. The current is again an inverse of

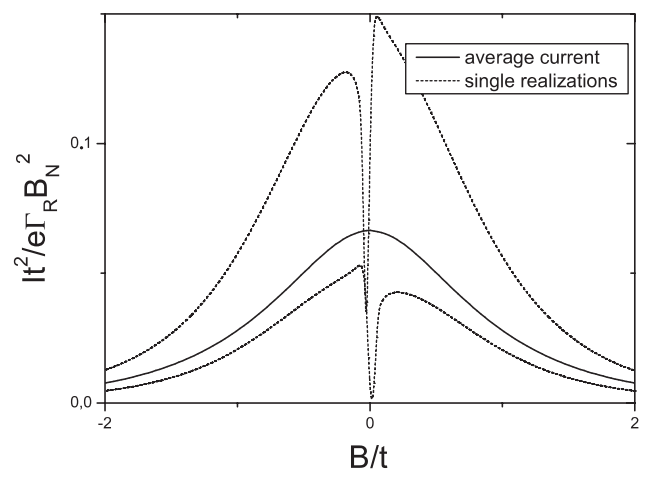

FIG. 2. Average current (solid line) and that for two random realizations of nuclear magnetic field in the limit of validity of (6) $\left(t / B_{N}=50, \Delta / t=1\right)$. Note stopping points at $B \simeq B_{N}$ seen for the realizations. 


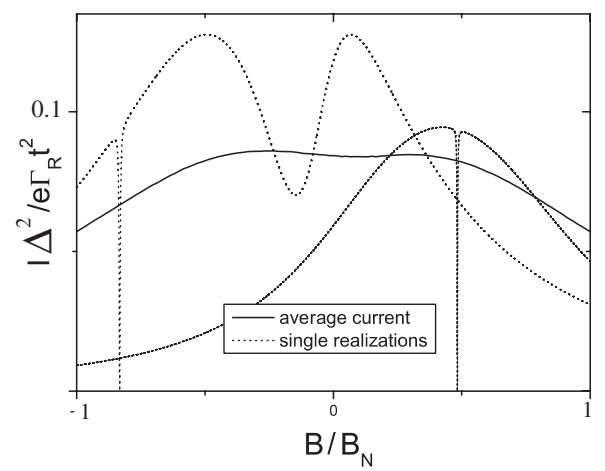

FIG. 3. Average current and that for two realizations in the limit of validity of (8) $\left(t / B_{N}=0.2, \Delta / t=-50\right)$. Note narrow dips of the current at stopping points.

the sum of inverse partial rates and reads

$$
I / e=\Gamma_{\text {in }}\left(\boldsymbol{n}_{L} \times \boldsymbol{n}_{R}\right)^{2} .
$$

The average current as a function of $B$ becomes

$$
\langle I\rangle / e=\Gamma_{\mathrm{in}} S\left(\sqrt{3} B / B_{N}\right) ;
$$

where

$$
\begin{aligned}
S(x) \equiv & 4 / x^{2}-6 / x^{4}+\sqrt{2 \pi} \operatorname{erfi}(x / \sqrt{2})\left(6 / x^{5}-2 / x^{3}\right) \\
& \times \exp \left(-x^{2} / 2\right)-3 \pi \operatorname{erfi}^{2}(x / \sqrt{2}) \exp \left(-x^{2}\right) / x^{6} .
\end{aligned}
$$

It is interesting to note a special form of this function: the graph of $S$ gives a peak with flat top, $S^{\prime \prime}(0)=0$. This function provides an excellent fit to experimental data (Fig. 4); those are impossible to fit with more conventional peak functions. Such flat peaks are thus specific for the model in use and provide strong support of its experimental validity.

In conclusion, we have presented the theoretical framework for the electron transport via a double quantum dot influenced and governed by nuclear magnetic field. Our approach is based on density matrix equations and we achieve good agreement with experiment [15] assuming averaging over realizations of nuclear fields. An important feature which is yet to be observed in the course of faster and more accurate measurement is the presence of stopping points for any given realization of nuclear fields. The width of the current dip near the stopping point is estimated as $\delta B \simeq B_{N}$ for $B_{N} \ll \Delta_{\mathrm{ST}}$ and $\delta B \simeq \Delta_{\mathrm{ST}}$ for $B_{N} \gg \Delta_{\mathrm{ST}}$.

If one interprets the effect of nuclear magnetic fields in terms of spin coherence time, the results of [15] are discouraging if not forbidding for QMC in GaAs quantum dot systems. The coherence time estimated is just too short, $\simeq 10^{-7} \mathrm{~s}$. We speculate that the presence of stopping points can remedy the situation. Faster current measurement

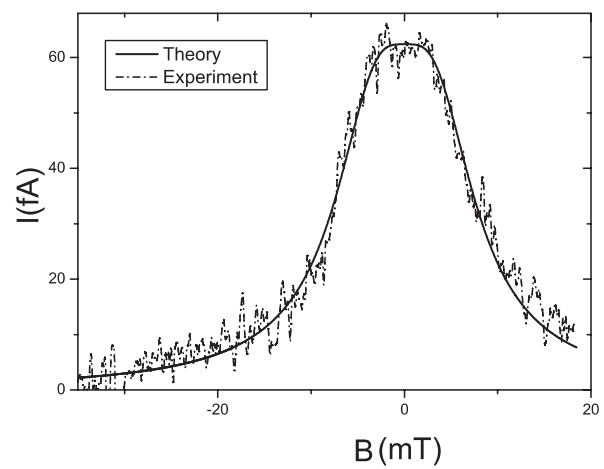

FIG. 4. Fit of experimental data [15] with "flat peak" relation (11) gives $B_{N}=4.75 \mathrm{mT}, \Gamma_{\text {in }}=0.63 \mathrm{MHz}$.

would allow us to characterize and, with the aid of external feedback, partially compensate the nuclear fields by stabilizing the system in the stopping point.

We are grateful to the authors of [15] for drawing our attention to the topic, many useful discussions, and communicating their results prior to publication. We acknowledge the financial support by FOM.

*Present address: Schlumberger Research and Development Inc., Taganskaya Street 9, Moscow, Russia.

[1] L. M. K. Vandersypen and I. L. Chuang, Rev. Mod. Phys. 76, 1037 (2005).

[2] Semiconductor Spintronics and Quantum Computation, edited by D. D. Awshalom, D. Loss, and N. Samarth (Springer-Verlag, Berlin, 2002).

[3] S. A. Wolf et al., Science 294, 1488 (2001).

[4] T. Fujisawa et al., Nature (London) 419, 278 (2002).

[5] R. Hanson et al., Phys. Rev. Lett. 91, 196802 (2003).

[6] K. Ono et al., Science 297, 1313 (2002).

[7] A. C. Johnson et al., Nature (London) 435, 925 (2005).

[8] D. Weinmann, W. Häusler, and B. Kramer, Phys. Rev. Lett. 74, 984 (1995).

[9] D. Paget, Phys. Rev. B 25, 4444 (1982).

[10] S.I. Erlingsson and Y. V. Nazarov, Phys. Rev. B 66, 155327 (2002).

[11] S. I. Erlingsson, Yu. V. Nazarov, and V.I. Fal'ko, Phys. Rev. B 64, 195306 (2001).

[12] K. Ono and S. Tarucha, Phys. Rev. Lett. 92, 256803 (2004).

[13] K. Hashimoto, K. Muraki, N. Kumada, T. Saku, and Y. Hirayama, Phys. Rev. Lett. 94, 146601 (2005).

[14] S. I. Erlingsson, O. N. Jouravlev, and Yu. V. Nazarov, Phys. Rev. B 72, 033301 (2005).

[15] F. Koppens et al., Science 309, 1346 (2005).

[16] A. V. Khaetskii and Y. V. Nazarov, Phys. Rev. B 61, 12639 (2000).

[17] T. H. Stoof and Yu. V. Nazarov, Phys. Rev. B 53, 1050 (1996). 